ESJ Natural/Life/Medical Sciences

\title{
Facteurs Associés à la Vaccination Complète chez les Enfants de 12 à 23 Mois en Guinée
}

\author{
M. Dilé Diallo, DESS \\ Direction de la Population, \\ Ministère du Plan et du Développement Economique, Conakry, Guinée
}

Doi:10.19044/esj.2021.v17n17p80

Submitted: 07 September 2020

Accepted: 27 April 2021

Published: 31 May 2021
Copyright 2021 Author(s)

Under Creative Commons BY-NC-ND

4.0 OPEN ACCESS

Cite As:

Diallo M.D. (2021). Facteurs Associés à la Vaccination Complète chez les Enfants de 12 à 23 Mois en Guinée. European Scientific Journal, ESJ, 17(17), 80.

https://doi.org/10.19044/esj.2021.v17n17p80

\section{Résumé}

La couverture vaccinale des enfants demeure faible et ne progresse qu'assez lentement en Guinée. En effet, les résultats de l'enquête démographique et de santé (EDS) 2018 indiquent que seulement $24 \%$ des enfants de 12-23 mois avaient reçu tous les vaccins de base recommandés par le programme élargi de vaccination (PEV) avant d'atteindre leur premier anniversaire. Environ $51 \%$ des enfants ont été partiellement vaccinés, et $22 \%$ des enfants de 12-23 mois n'ont reçu aucun vaccin. Les variations selon les régions de résidence sont importantes et mettent en évidence la faiblesse de la couverture vaccinale en milieu rural et dans les régions de Labé, Mamou et Kindia. L'objectif de cette étude était de déterminer les facteurs associés à la vaccination complète chez les enfants 12-23 mois. Avec les données de l'EDS 2018, on a constaté que les principaux déterminants de la vaccination chez les enfants étaient le niveau d'instruction de la mère et du père, l'accouchement dans un établissement de santé, davantage de consultations prénatales, le fait d'appartenir à un ménage de taille plus réduite, la région de résidence et le niveau de vie du ménage. Les résultats obtenus peuvent servir à l'amélioration des stratégies de mobilisation sociale pour l'augmentation de la couverture vaccinale des enfants, notamment par la sensibilisation des communautés sur l'intérêt de la vaccination des enfants, la formation du personnel de santé en counseling afin de réduire les déperditions et favoriser le respect du calendrier 
de vaccination.

Mots clés : Guinée, vaccination des enfants, soins préventifs, enfants de 1223 mois

\title{
Factors Associated with Complete Vaccination in Children 12 to 23 Months in Guinea
}

\author{
M. Dilé Diallo, DESS \\ Direction de la Population, \\ Ministère du Plan et du Développement Economique, Conakry, Guinée
}

\begin{abstract}
Childhood immunization coverage remains low and is progressing rather slowly in Guinea. Indeed, the results of the 2018 DHS indicate that only $24 \%$ of children aged 12-23 months had received all basic EPI vaccines before reaching their first birthday. About $51 \%$ of children were partially immunized, and $22 \%$ of children $12-23$ months did not receive any vaccine. The variations according to the regions of residence are significant and highlight the low level of vaccination coverage in rural areas and in the regions of Labé, Mamou and Kindia. The objective of this study was to determine the factors associated with complete vaccination in children 12-23 months. With data from the DHS 2018 , it was found that the main determinants of vaccination in children were the level of education of the mother and father, delivery in a health facility, more antenatal consultations, belonging to a smaller household, region of residence and wealth quintile. The results obtained can be used for the development of social mobilization strategies to increase the immunization coverage of children, in particular by sensitizing communities on the value of immunization of mothers and children, training of health personnel. in counseling to reduce wastage and meet vaccination deadlines.
\end{abstract}

Keywords: Guinea, Childhood immunization, preventive care, children 12-23 months

\section{Introduction}

En Afrique subsaharienne, près de 31 millions d'enfants de moins de 5 ans souffrent chaque année de maladies évitables par la vaccination. Plus d'un demi-million d'entre eux meurent faute d'accès aux vaccins dont ils avaient besoin (OMS, 2019). La probabilité qu'un enfant né aujourd'hui soit complètement vacciné avec tous les vaccins recommandés au niveau mondial avant d'atteindre l'âge de 5 ans est inférieure à $20 \%$ (UN, 2020). En 2018, les 
Chefs d'Etat et de Gouvernement africains ont adopté la Déclaration d'Addis Abeba sur la vaccination (DAV), pour renouveler leur engagement d'ouvrer à la vaccination de tous les enfants.

La plus grande partie des décès observés parmi les enfants de moins 5 ans dans les pays en développement sont dus à un nombre restreint de maladies infectieuses et parasitaires évitables par la vaccination (Akoto et Tabutin, 1985 ; Pison et al., 1988 ; Barbieri, 1991). Grâce à la vaccination la variole a été éradiquée et la poliomyélite est sur le point d'être vaincue. L'impact des vaccins ne se limite pas seulement à la santé publique ; ils ont également une incidence sur la performance scolaire des enfants, sur l'augmentation des revenus des ménages et, en définitive, sur la croissance économique des pays (OMS, 2017).

Pour assurer une protection maximale aux enfants, l'OMS et l'UNICEF recommandent aux Etats un certain nombre de vaccins suivant un calendrier précis pour éradiquer les six maladies infantiles courantes qui peuvent être prévenues par la vaccination à savoir : la tuberculose, la diphtérie, la coqueluche, le tétanos, la poliomyélite et la rougeole. Ainsi, un enfant est considéré complètement vacciné ${ }^{1}$ que lorsqu'il a reçu le BCG (protection contre la tuberculose), les trois doses de vaccin contre la polio, trois doses de DTCoq (Diphtérie, Tétanos et Coqueluche) et le vaccin contre la rougeole. Tous ces vaccins doivent être complétés pour tous les enfants avant leur premier anniversaire (OMS, 2017). En outre, l'OMS a recommandé en 2012, à tous les Etats une couverture vaccinale en fin 2020 de $90 \%$ au niveau national et de $80 \%$ dans chaque district sanitaire pour tous les vaccins du programme national de vaccination afin de faire bénéficier à tous les avantages de la vaccination. Malheureusement ces cibles sont loin d'être atteintes encore dans des nombreux pays africains.

En Guinée, la couverture vaccinale demeure faible et ne progresse pas. Les résultats de l'enquête démographique et de santé (EDS) 2018 indiquent que seulement $24 \%$ des enfants de 12-23 mois avaient reçu tous les vaccins de base du PEV dont $19 \%$ les avaient reçus à l'âge approprié, c'est-à-dire avant l'âge de 12 mois. Plus de la moitié des enfants (51\%) n'ont été que partiellement vaccinés. Les disparités entre les milieux et régions de résidence sont importantes. En milieu urbain un tiers des enfants sont complètement vaccinés contre un quart en milieu rural $(21 \%)$. Selon les régions le pourcentage d'enfants complètement vaccinés varie de $8 \%$ à Labé à $36 \%$ à Kankan. Même à Conakry, la capitale, le niveau de couverture vaccinale complète n'est pas élevé ( $37 \%$ ). En outre, $12 \%$ des enfants de 12-23 mois n'ont reçu aucun vaccin en 2018. Les déperditions entre les doses de vaccins

En Guinée, le carnet de vaccination du PEV comprend le BCG et polio 0 à la naissance, Penta1 et Polio- 1 dans la $6^{\text {ème }}$ semaine, Penta- 2 et Polio- 2 dans la $10^{\text {ème }}$ semaine, Penta-3 et Polio3 dans la $14^{\text {ème }}$ semaine, rougeole-1 à 9 mois. 
sont importantes. La couverture pour la première dose de pentavalent passe de $62 \%$ à $40 \%$ pour la troisième. Pour la Polio, la déperdition est plus importante passant de $65 \%$ pour la première à $40 \%$ pour la troisième.

En outre, la mortalité des enfants reste élevée en Guinée même si une tendance à la baisse est notée au cours des 20 dernières années. Entre 1999 et 2018 le niveau de la mortalité des enfants de moins de 5 ans a baissé de $37 \%$. Cette baisse est principalement due à la mortalité juvénile (1 à 4 ans). La mortalité infantile (néonatale et post-néonatale) est restée constante au cours des cinq dernières années (EDS, 2018).

Bien que de nombreuses études ont étudié les déterminants sociodémographiques de la vaccination en Afrique, rares sont celles qui ont étudié le cas guinéen. Cette étude a pour objectif de palier à cette insuffisance, ainsi d'identifier les facteurs associés à la vaccination complète des enfants âgés de 12 à 23 mois en Guinée.

L'étude s'articule autour de trois sections. La première traite des données et aspects méthodologiques. La deuxième section présente les résultats des analyses descriptive et explicative, et enfin une discussion et conclusion clôture le papier.

\section{Méthodologie}

Cette étude est faite à partir des données de l'enquête démographique et de santé (EDS) réalisée en Guinée, en 2018. Ces données contiennent pour chaque enfant né au cours des 5 dernières années, les informations relatives à la grossesse, aux soins prénatals et postnatals, à la vaccination, etc., ainsi que les caractéristiques sociodémographiques de la mère de chacun de ces enfants. La population cible de l'étude est constituée des enfants âgés de 12 à 23 mois au moment de l'enquête. C'est cette tranche d'âge qui est recommandée par l'OMS dans la mesure de la couverture vaccinale afin de minimiser les biais de mémoire (OMS, 2012).

Le statut vaccinal de l'enfant constitue la variable dépendante. Elle comprend trois modalités : non vacciné, partiellement vacciné, complètement vacciné. L'enfant complètement vacciné est celui qui a reçu le BCG (vaccin contre la tuberculose), les trois doses de vaccin contre la poliomyélite, les trois doses de diphtérie, tétanos, coqueluche (DTCoq ou vaccin pentavalent) et le vaccin contre la rougeole sur la base du carnet de vaccination ou de la déclaration de la mère.

Les variables indépendantes sont composées des caractéristiques de l'enfant, de sa mère et du ménage.

- Caractéristiques de l'enfant : le sexe, le rang de naissance $\left(1^{\text {er }}, 2^{\text {ème }}\right.$, $3^{\text {ème }}, 4^{\mathrm{ème}}$ ou plus), le lien de parenté avec le chef de ménage.

- Caractéristiques de la mère : l'âge de la mère à l'enquête, le niveau d'instruction de la mère, l'état matrimonial de la mère, le fait d'exercer 
une activité économique, l'autonomie de la mère en matière de recours aux soins de santé, le lieu d'accouchement, le nombre de consultations prénatals durant la dernière grossesse.

- Caractéristiques liées au ménage : la taille du ménage, le nombre d'enfants de moins de 5 ans; Le milieu et la région de résidence, le niveau de vie du ménage est une variable composite construit à partir des biens possédés et des commodités de logement.

- Le choix de ces variables est basé sur la revue de la littérature, le contexte de l'étude et les variables disponibles dans le fichier des données.

L'analyse des données a été faite en deux étapes. Dans un premier temps, l'analyse bivariée a été effectuée pour évaluer l'association entre les variables indépendantes et la variable dépendante à l'aide du test de chi carré (tableau 1). En deuxième lieu, une régression logistique multinomiale a été utilisée pour déterminer la signification des facteurs liés au statut vaccinal après contrôle des autres variables indépendantes.

La régression logistique multinomiale est une méthode d'analyse explicative adaptée aux situations où les variables dépendantes sont qualitatives et polytomiques. C'est une extension de la régression logistique binaire aux variables qualitatives à trois modalités ou plus. Pour utiliser le Logit multinomial, il faut nécessairement que les variables dépendantes soient uniques et homogènes ; c'est à dire que leurs modalités soient exclusives. Il se présente des situations différentes selon que la variable explicative à introduire dans le modèle est qualitative ou continue. Au cas où elle est qualitative, on choisit une modalité de référence par rapport à la quelle sera interprété les résultats (traduits ici en termes de risque). Ces risques se calculent à l'aide de l'exponentiel des coefficients.

Les résultats de la régression logistique multinomiale sont présentés au tableau 2 pour le rapport de risque relatif ajusté (RRR) et l'intervalle de confiance (IC) à $95 \%$. Toute valeur de probabilité inférieure à 5\% a été considérée comme statistiquement significative. Les estimations ont été pondérées pour représenter la population au niveau national, et l'effet d'un plan d'échantillonnage complexe à plusieurs degrés a été pris en compte dans l'analyse. 


\section{Résultats}

Il s'agit dans cette section de décrire les caractéristiques des enfants, de leurs mères et des ménages dans lesquels ils vivent selon le statut vaccinal de l'enfant. Dans un premier temps, on examine la prévalence et les variations des vaccinations selon les différentes caractéristiques et, dans un deuxième temps, on procède à l'analyse multivariée à l'aide de la régression logistique multinomiale pour cerner les déterminants principaux de la vaccination des enfants de 12-23 mois en Guinée.

\section{Résultats descriptifs}

Le tableau 1 présente le croisement entre le statut vaccinal et les caractéristiques de l'enfant, de la mère, du chef de ménage et du ménage. Au seuil de 5\%, on ne constate aucune différence significative dans la vaccination selon les caractéristiques propres à l'enfant (sexe, rang de naissance et lien de parenté avec le chef de ménage). Par contre le statut vaccinal de l'enfant est associé significativement avec les caractéristiques de la mère (exceptées l'âge et l'état matrimonial), et celles du ménage.

La prévalence de la vaccination complète était plus faible parmi les enfants dont la mère n'avait fait aucune consultation prénatale durant la grossesse (10\%), parmi les enfants nés à domicile (13\%), et parmi ceux dont le mari/conjoint de la femme est celui qui décide seul des soins de santé (17\%) ; les enfants dont le mari/conjoint est sans instruction (20\%). Enfin, plus la taille du ménage augmente, plus la proportion d'enfants complètement vaccinés diminue. La couverture vaccinale est plus faible chez les enfants issus des ménages classés plus pauvres comparativement aux enfants des ménages aisés $(15 \%$ contre $36 \%)$. La vaccination complète était plus faible dans la région de Labé (8\%), suivi de Kindia (11\%) et de Mamou (13\%).

Tableau 1 : Proportion d'enfants de 12-23 mois par caractéristiques sociodémographiques des enfants, des mères et des ménages

\begin{tabular}{|c|c|c|c|c|c|}
\hline \multirow[b]{2}{*}{ Variables } & \multicolumn{5}{|c|}{ Statut } \\
\hline & $\begin{array}{c}\text { Non } \\
\text { vacciné }\end{array}$ & $\begin{array}{l}\text { Partiellement } \\
\text { vacciné }\end{array}$ & $\begin{array}{l}\text { Complètement } \\
\text { vacciné }\end{array}$ & Effectif & $\begin{array}{l}\text { Prob } \\
\text { khi2 }\end{array}$ \\
\hline \multicolumn{6}{|c|}{ Caractéristiques des enfants } \\
\hline \multicolumn{5}{|c|}{ Sexe enfant } & 0,471 \\
\hline Masculin & 22,2 & 52,4 & 25,4 & 727 & \\
\hline Féminin & 22,7 & 55,0 & 22,2 & 657 & \\
\hline \multicolumn{5}{|l|}{ Rang de naissance } & 0,136 \\
\hline 1 & 19,5 & 50,8 & 29,7 & 275 & \\
\hline $2-3$ & 20,7 & 56,1 & 23,2 & 531 & \\
\hline $4-5$ & 25,6 & 51,2 & 23,2 & 358 & \\
\hline $6+$ & 25,4 & 55,3 & 19,3 & 220 & \\
\hline \multicolumn{5}{|c|}{ Lien de parenté avec le chef ménage } & 0,579 \\
\hline Fils, fille & 22,9 & 54,1 & 22,9 & 1,087 & \\
\hline Petit fils/fille & 24,5 & 46,4 & 29,1 & 109 & \\
\hline Autre parent & 17,0 & 55,8 & 27,2 & 119 & \\
\hline Adopté/Sans lien & 21,4 & 53,5 & 25,1 & 69 & \\
\hline
\end{tabular}




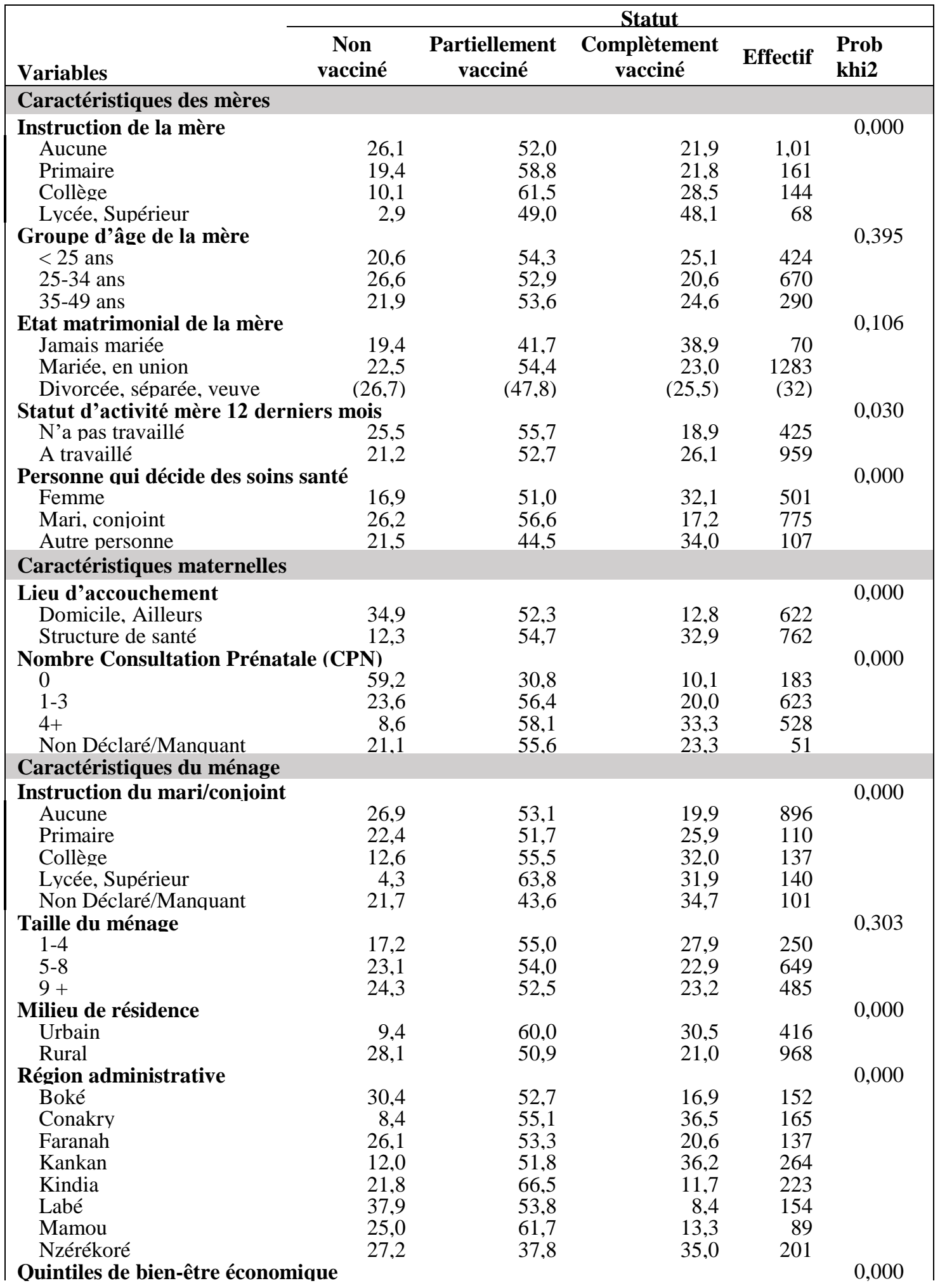




\begin{tabular}{|c|c|c|c|c|c|}
\hline \multirow[b]{2}{*}{ Variables } & \multicolumn{5}{|c|}{ Statut } \\
\hline & $\begin{array}{c}\text { Non } \\
\text { vacciné }\end{array}$ & $\begin{array}{l}\text { Partiellement } \\
\text { vacciné }\end{array}$ & $\begin{array}{l}\text { Complètement } \\
\text { vacciné }\end{array}$ & Effectif & $\begin{array}{l}\text { Prob } \\
\text { khi2 }\end{array}$ \\
\hline Le plus bas & 38,1 & 46,7 & 15,2 & 309 & \\
\hline Second & 25,9 & 52,2 & 21,8 & 324 & \\
\hline Moyen & 24,3 & 53,1 & 22,6 & 258 & \\
\hline Quatrième & 10,7 & 62,9 & 26,4 & 249 & \\
\hline Le plus élevé & 8,1 & 55,5 & 36,3 & 244 & \\
\hline Ensemble & 22,5 & 53,6 & 23,9 & 1384 & \\
\hline
\end{tabular}

() basé sur un effectif de moins de 50 cas non pondérés ; *** $: \leq \mathrm{p} 0,01 ; * *: \leq \mathrm{p} 0,05 ; *: \leq \mathrm{p} 0,10$

\section{Résultats explicatifs}

Le les résultats de la régression logistique multinomiale de la vaccination des enfants de 12-23 mois (tableau 2) montre que les caractéristiques qui sont associés au risque relatif de vaccination (complète ou partielle) des enfants sont : le niveau d'instruction de la mère, le lieu d'accouchement, le nombre de consultations prénatales, le niveau d'instruction du père, la taille du ménage, la région de résidence et le niveau de vie du ménage.

Le niveau d'instruction de la mère est l'un des déterminants principaux du recours aux soins de santé. Les enfants dont la mère a un niveau d'instruction supérieur au collège, ont 4,18 plus de chance d'être complètement vaccinés que d'être non vaccinés ( $R R R=4,18$, IC $95 \%=0,78$ 22,36). Il n'y a pas de différence significative entre les enfants partiellement vaccinés et ceux non vaccinés comparativement au niveau d'instruction de la mère.

Le lieu de l'accouchement influence de façon significative les chances de l'enfant d'être vacciné. En effet, les enfants nés dans un établissement de santé par rapport à ceux qui sont nés à domicile ont 2,7 fois plus de chances d'être complètement vaccinés par rapport aux non-vaccinés $(\mathrm{RRR}=2,7$, IC $95 \%=1,7-4,4)$. Pour les enfants partiellement vaccinés cette possibilité était de 1,5 fois plus élevée par rapport aux enfants non-vaccinés $(\mathrm{RRR}=1,5$, IC $95 \%=1,0-2,1)$.

Les enfants dont les mères ont effectué 1 à 3 consultations prénatales avaient 2,9 fois plus de chances d'être complètement vacciné par rapport aux non-vaccinés (RRR =2,9, IC 95\% = 1,3-6,7), et cette chance était de 3,9 fois plus élevée d'être partiellement vacciné toujours par rapport aux non vaccinés (RRR = 3,9, IC 95\% = 2,4-6,2). Quand la mère a effectué quatre CPN ou plus les chances de l'enfant d'être complètement ou partiellement vacciné sont multipliées respectivement par $12(\mathrm{RRR}=11,6$, IC 95\% = 4,7-26,2) et par 8 $(\mathrm{RRR}=8,3$, IC 95\% = 4,7-14,5).

Le niveau d'instruction du père s'est révélé également très discriminant pour la vaccination complète des enfants. Les enfants dont le père 
avait un niveau d'instruction secondaire ou plus avaient 2 fois plus de chances d'être complètement vaccinés que les enfants dont le père est sans instruction (RRR $=1,9$, IC 95\% = 1,0-3,4). Il n'y avait pas de différence significative entre les enfants partiellement vaccinés par rapport au niveau d'instruction du père. Les enfants issus des ménages de petite taille ( 1 à 4 personnes) avaient 2,6 fois plus de chances d'être complétement vaccinés comparé aux enfants issus des ménages ayant 9 membres ou plus (RRR = 2,6, IC 95\% = 1,2-5,4). Ces chances sont 1,7 fois plus grandes pour l'enfant d'être partiellement vacciné s'il est issu d'un ménage de 1 à 4 personnes que s'il appartenait à un ménage de 9 personnes ou plus $(\mathrm{RRR}=1,7$, IC $95 \%=0,9-3,0)$.

Par rapport à la région administrative, les résultats montrent que comparés aux enfants non vaccinés, les enfants de la région de Kankan avaient 13,6 fois plus de chance de recevoir tous les vaccins de base que ceux de la région de Labé. Dans les régions Faranah et de Nzérékoré, ce risque était respectivement de 5,8 et 5,6 fois; tandis que dans les régions de Boké et de Mamou ce risque était de 2,7 et 2,6 fois plus élevé. Cependant, pour les enfants partiellement vaccinés, seuls les enfants de la région de Kankan présentaient une chance plus élevée d'être partiellement vacciné que les enfants de Labé $(\mathrm{RRR}=2,6$, IC 95\% = 1,1-5,9).

Les enfants appartenant aux dernières catégories de ménages les plus aisés ont respectivement 2,5 fois et 3,9 fois plus de chance d'être complètement vaccinés que d'être non vaccinés. Les enfants des ménages riches (quatrième quintile) ont 2 fois plus de chances d'être partiellement vaccinés que d'être non vaccinés.

Tableau 1 : Résultats de la régression logistique multinomiale de la vaccination des enfants de 12-23 mois en Guinée

\begin{tabular}{|c|c|c|c|c|}
\hline \multirow[b]{2}{*}{ Variables } & \multicolumn{2}{|c|}{ Complètement vacciné } & \multicolumn{2}{|c|}{ Partiellement vacciné } \\
\hline & RRR & $95 \%$ IC & RRR & $95 \%$ IC \\
\hline \multicolumn{5}{|c|}{ Caractéristiques des enfants } \\
\hline \multicolumn{5}{|c|}{ Sexe enfant } \\
\hline Masculin (Ref.) & & & & \\
\hline Féminin & 0,85 & $0,572-1,269$ & 0,988 & $0,724-$ \\
\hline \multicolumn{5}{|l|}{ Rang de naissance } \\
\hline & 1,26 & $0,496-3,222$ & 1,009 & $0,450-$ \\
\hline $2-3$ & 0,95 & $0,458-1,985$ & 1,071 & 0,609 \\
\hline $4-5$ & 1,04 & $0,514-2,105$ & 0,86 & $0,506-$ \\
\hline \multirow{2}{*}{\multicolumn{5}{|c|}{ Lien de parenté avec le chef ménage }} \\
\hline & & & & \\
\hline \multicolumn{5}{|c|}{ Fils,fille (Ref.) } \\
\hline Petit fils/fille & 0,97 & $0,394-2,417$ & 0,75 & $0,370-$ \\
\hline Autre parent & 1,61 & $0,708-3,678$ & 1,253 & $0,610-$ \\
\hline Adopté/Non apparenté & 1,52 & $0,664-3,522$ & 1,28 & $0,654-$ \\
\hline \multicolumn{5}{|c|}{ Caractéristiques des mères } \\
\hline \multirow{2}{*}{\multicolumn{5}{|c|}{$\begin{array}{l}\text { Instruction de la femme } \\
\text { Aucune (Ref.) }\end{array}$}} \\
\hline Aucune (Ref.) & & & & \\
\hline Primaire & 0,86 & $0,468-1,595$ & 1,052 & $0,635-$ \\
\hline Collège & 1,24 & $0,541-2,885$ & 1,415 & $0,720-$ \\
\hline
\end{tabular}




\begin{tabular}{|c|c|c|c|c|}
\hline \multirow{2}{*}{ Variables } & \multicolumn{2}{|c|}{ Complètement vacciné } & \multicolumn{2}{|c|}{ Partiellement vacciné } \\
\hline & RRR & $95 \%$ IC & RRR & $95 \%$ IC \\
\hline Lycée, Supérieur & $4,14^{*}$ & $0,776-22,12$ & 2,507 & $0,486-$ \\
\hline \multicolumn{5}{|c|}{ Groupe d'âge de la femme } \\
\hline$<25$ ans & 0,98 & $0,467-2,067$ & 0,93 & $0,483-$ \\
\hline $25-34$ ans & 1,15 & $0,671-1,977$ & 0,98 & $0,642-$ \\
\hline \multicolumn{5}{|l|}{ 35-49 ans (Ref.) } \\
\hline \multicolumn{5}{|c|}{ Etat matrimonial de la femme } \\
\hline Jamais mariée & 1,33 & $0,336-5,305$ & 1,082 & $0,351-$ \\
\hline Mariée, union & 1,48 & $0,105-21,04$ & 1,934 & $0,245-$ \\
\hline \multicolumn{5}{|c|}{ Divorcée, séparée, veuve (Ref.) } \\
\hline \multicolumn{5}{|c|}{ Statut d'activité mère 12 derniers mois } \\
\hline \multicolumn{5}{|c|}{ N'a pas travaillé (Ref.) } \\
\hline A travaillé & 1,47 & $0,905-2,379$ & 1,194 & $0,814-$ \\
\hline \multicolumn{5}{|c|}{ Personne qui décide des soins sante } \\
\hline Femme (Ref.) & 1,15 & $0,104-12,76$ & 0,674 & $0,103-$ \\
\hline Mari, partenaire & 0,58 & $0,0551-$ & 0,58 & $0,0936-$ \\
\hline Autre personne & & & & \\
\hline \multicolumn{5}{|c|}{ Caractéristiques maternelles } \\
\hline \multicolumn{5}{|c|}{ Lieu d'accouchement } \\
\hline \multicolumn{5}{|c|}{ Domicile, Ailleurs (Ref.) } \\
\hline Structure de santé & $2,69 * * *$ & $1,675-4,343$ & $1,419 *$ & $0,976-$ \\
\hline \multicolumn{5}{|c|}{ Nombre Consultation Prénatale } \\
\hline \multicolumn{5}{|c|}{ Aucun (Ref.) } \\
\hline $1-3$ & $2,89 * * *$ & $1,302-6,450$ & $4,025 * * *$ & $2,493-$ \\
\hline $4+$ & $11,00 * * *$ & $4,547-26,62$ & $8,497 * * *$ & $4,870-$ \\
\hline Non déclaré & $2,88 *$ & $0,887-9,380$ & $3,882 * * *$ & $1,745-$ \\
\hline \multicolumn{5}{|c|}{ Caractéristiques des ménages } \\
\hline \multicolumn{5}{|c|}{ Instruction du mari/conjoint } \\
\hline \multicolumn{5}{|c|}{ Aucun (Ref.) } \\
\hline Primaire & 0,91 & $0,452-1,830$ & 0,784 & $0,451-$ \\
\hline Collège & 1,73 & $0,834-3,604$ & 1,38 & $0,699-$ \\
\hline Lycée, Supérieur & $2,43 *$ & $0,924-6,387$ & $3,474 * * *$ & $1,398-$ \\
\hline Manquant & $(-)$ & $(-)$ & & $(-)$ \\
\hline \multicolumn{5}{|l|}{ Taille du ménage } \\
\hline $1-4$ & $2,70 * * *$ & $1,302-5,614$ & $1,717^{*}$ & $0,951-$ \\
\hline $5-8$ & 1,37 & $0,856-2,190$ & 1,19 & $0,812-$ \\
\hline \multicolumn{5}{|l|}{$9+($ Ref.) } \\
\hline \multicolumn{5}{|l|}{ Milieu de résidence } \\
\hline Urbain & 0,70 & $0,269-1,820$ & 1,346 & $0,643-$ \\
\hline Rural (Ref.) & & & & \\
\hline \multicolumn{5}{|c|}{ Région administrative } \\
\hline Boké & $2,803 * *$ & $1,078-7,290$ & 1,157 & $0,612-$ \\
\hline Conakry & $3,956 * *$ & $1,193-13,12$ & 1,18 & $0,441-$ \\
\hline Faranah & $5,979 * * *$ & $2,070-17,27$ & 1,70 & $0,861-$ \\
\hline Kankan & $13,93 * * *$ & $4,283-45,31$ & $2,672 * *$ & $1,193-$ \\
\hline Kindia & 1,53 & $0,603-3,902$ & 1,45 & $0,767-$ \\
\hline Labé (Ref.) & & & & \\
\hline Mamou & $2,652 *$ & $0,877-8,019$ & 1,71 & $0,757-$ \\
\hline Nzerekore & $5,696 * * *$ & $2,178-14,90$ & 0,88 & $0,415-$ \\
\hline
\end{tabular}




\begin{tabular}{|c|c|c|c|c|}
\hline \multirow[b]{2}{*}{ Variables } & \multicolumn{2}{|c|}{ Complètement vacciné } & \multicolumn{2}{|c|}{ Partiellement vacciné } \\
\hline & RRR & $95 \%$ IC & RRR & $95 \%$ IC \\
\hline \multicolumn{5}{|c|}{ Niveau de vie du ménage } \\
\hline \multicolumn{5}{|c|}{ Très pauvre (Ref.) } \\
\hline Pauvre & 1,45 & $0,797-2,626$ & 1,298 & $0,850-$ \\
\hline Moyen & 1,37 & $0,688-2,728$ & 1,30 & $0,845-$ \\
\hline Riche & $2,58 *$ & $0,982-6,789$ & $2,003^{*}$ & $0,967-$ \\
\hline Très riche & $3,99 * *$ & $1,068-14,92$ & 1,59 & $0,541-$ \\
\hline \multicolumn{5}{|c|}{$\begin{array}{l}\mathrm{RRR}=\text { Relative risk ratio } ; \mathrm{IC}=\text { Intervalle de confiance } \\
* \mathrm{p} \text { value }<0.1, * * \mathrm{p} \text { value }<0.05, * * * \mathrm{p} \text { value }<0.01 \\
(-) \text { valeur omise, n'a pas été déclarée dans le tableau }\end{array}$} \\
\hline
\end{tabular}

\section{Discussion}

Il apparait des résultats que les caractéristiques de l'enfant ne sont pas associées avec une plus grande chance de vaccination de l'enfant en Guinée. Un constat similaire a été fait par Aalemi et al. (2019), Tefera et al. (2018); Xeuatvongsa et al. (2017). Cependant, certains auteurs postulent que très souvent, la préférence pour les enfants de sexe masculins dans certaines sociétés conduit à une faible attention pour les soins des filles (Locoh, 1989). Dans une étude faite à Diourbel (Sénégal) Ndèye et al. (2009) concluent que la chance d'être complètement vaccinés était plus élevée quand l'enfant était un garçon. Par contre Baya (1996), dans une étude sur le comportement des mères en matière de Santé à Bobo-Dioulasso (Burkina Faso), a montré que contrairement aux attentes, les filles avaient plus de chance d'être vacciné contre la rougeole que les garçons. Un résultat similaire a été trouvé à Madagascar par Rakondrabe (2004).

Le rang de naissance ne s'est pas avéré discriminant pour la vaccination complète ou partielle des enfants. Alors que plusieurs études ont indiqué que plus le rang de naissance est élevé, moins le recours aux services de santé est accru. Sawadogo (2008) a montré que contrairement aux enfants premiers nés, les enfants de rang élevés bénéficient généralement de soins de moindre qualité ; l'attention accordée à l'enfant par la mère diminuant au fur et à mesure que le rang augmente. Aussi, Mekonnen et Mekonnen (2002) en Éthiopie ont souligné dans leurs études que la probabilité qu'une femme reçoive des soins prénatals diminue au fur et à mesure que le nombre d'enfants augmente. Selon ces auteurs, lorsque les femmes ont un certain nombre d'enfants, elles considèrent qu'elles ont acquis une certaine connaissance et expérience de la maternité ; par conséquent, elles n'éprouvent pas tellement le besoin d'aller faire des consultations prénatales.

Le niveau d'instruction de la mère influence les chances de l'enfant d'être vacciné. Mais les différences ne se révèlent significatives que pour les enfants dont la mère a un niveau d'instruction supérieur au collège. L'instruction des parents, notamment de la mère a été identifiée partout 
comme l'un des facteurs les plus déterminants dans le recours aux soins de santé moderne. Mbacké et Van de Walle (1987) ont démontré dans une étude sur l'Afrique de l'Ouest que les femmes instruites portent beaucoup d'intérêt aux soins préventifs et recourent plus facilement aux services de santé lorsque leurs enfants tombent malades. L'instruction de la mère influence positivement le recours sanitaire, notamment à la vaccination (Zoungrana, 1993 ; Baya, 1998). Pour Fournier et Haddad (1995), l'amélioration du niveau d'instruction des populations entraine un renforcement de l'utilisation des soins de santé modernes. Également, Douba et al. (2015) dans une analyse portant sur des données du Burkina Faso, de la Côte d'Ivoire, du Ghana et de la Guinée concluent que les enfants des mères analphabètes avaient un risque élevé d'être incomplètement vaccinés comparés à ceux des mères alphabètes.

Le niveau d'instruction du père apparaît également comme un déterminant dans la vaccination des enfants. Et comme pour la mère, les effets de l'instruction du père ne se font sentir qu'à partir du deuxième cycle du secondaire tant pour la vaccination complète que partielle. Ce résultat confirme le rôle important de l'éducation sur le recours aux soins de santé.

Contrairement à ce qui a été attendu, le groupe d'âge de la mère n'influence pas de façon significative la vaccination des enfants en Guinée. Cependant, des études réalisées au Mali (Zoungrana, 1993), au Burkina Faso (Baya, 1996), et à Madagascar (Rakotodranbé, 2004) ont montré que les enfants des mères âgées avaient plus de chance de recevoir tous les vaccins requis.

Les enfants nés à domicile avaient un risque plus élevé d'être non vaccinés comparés à ceux nés dans une structure de santé. Des études précédentes réalisées au Bénin, au Kenya, et en Ouganda avaient aussi trouvé que la naissance à domicile constituait un facteur de risque de vaccination incomplète des enfants. Selon Douba et al. (2015), l'association entre l'accouchement à domicile et la vaccination incomplète des enfants pourrait s'expliquer par la persistance des facteurs ayant occasionné l'accouchement en dehors d'un centre de santé. En effet, après l'accouchement, si les facteurs ayant entraîné la naissance des enfants à domicile demeurent, les mères ne fréquentent pas ou fréquentent rarement des centres de santé pour des soins préventifs y compris la vaccination qui nécessite cinq visites d'un centre de vaccination pour que l'enfant reçoive la totalité des vaccins du PEV de routine.

Le nombre de consultations prénatales (CPN) semble être la variable la plus corrélée avec la vaccination des enfants de 12-23 mois en Guinée. En effet, les chances d'un enfant d'être complètement vacciné sont trois fois plus élevées si sa mère a fait 1 à $3 \mathrm{CPN}$, et 12 fois si la mère a fait plus de quatre visites prénatales. On sait que les $\mathrm{CPN}$ sont une occasion pour la future mère de recevoir des conseils de la part du personnel de santé sur les suites de la grossesse, l'accouchement et les soins au nouveau-né y compris la 
vaccination. L'influence positive de la CPN sur la vaccination complète des enfants a été attestée par Sia (2010) au Burkina Faso, Aalami et al. (2019) en Afghanistan et Dixit et al. (2013) en Inde.

Dans certaines sociétés le statut de la femme ne favorise pas son accès aux soins de santé moderne. La femme pour qu'elle puisse se rendre dans une structure de santé pour ses soins ou pour les soins de son enfant a besoin de l'autorisation du mari ou du chef de ménage. Les résultats montrent qu'il n'y a pas d'association significative entre la vaccination des enfants et l'autonomie de la femme dans la prise de décision pour les soins de santé. Il en est de même pour l'activité économique. Le fait d'exercer une activité au cours des 12 derniers mois n'influence pas la vaccination complète des enfants.

La taille du ménage influence négativement les chances de l'enfant d'être vacciné. Les enfants issus des ménages de petites taille ( 1 à 4 personnes) ont nettement plus de chance d'être vaccinés que ceux des ménages de taille plus grande. Ce résultat s'expliquerait aussi par le fait que les ménages de petite taille sont dirigés par des personnes plus instruites. Selon Akoto et al. (2002), plus que la taille du ménage, c'est le lien de parenté entre l'individu et le chef de ménage qui détermine la célérité du recours aux soins de santé moderne. Ce recours dépend du type de maladie et du statut du malade dans le ménage. Par conséquent, le respect des rendez-vous pour la vaccination peut varier en fonction du lien de parenté avec le chef de ménage. Gage et al. (1997) trouvent que vivre dans une famille élargie au Nigeria conférait à l'enfant un avantage pour son statut vaccinal. Ils attribuent cette différence à la capacité financière qui est à l'avantage des familles élargies. Dans la même étude, ils ne notent aucun impact de la structure du ménage sur le statut vaccinal au Niger.

En Afrique sub-saharienne, le milieu de résidence est fortement associé à l'utilisation des services de santé, indiquant ainsi que la différence dans le recours aux soins préventifs et curatifs est dû à une difficulté d'accès aux structures de santé. Des études faites au Ghana (Addai, 2000), en Ethiopie (Mekonnen et al., 2002) et au Burkina Faso (Banque mondiale, 2003), ont montré que les femmes qui vivent dans des les zones rurales ont un accès très limité aux structures sanitaires. Les raisons évoquées pour justifier cet état de fait sont que les services de santé ne sont pas disponibles, sont inaccessibles ou sont de qualité médiocre. A celles-ci s'ajoutent également l'analphabétisme des populations, la pauvreté, l'éloignement des établissements de santé, et la méconnaissance des parents sur l'importance de la vaccination dans les zones rurales (Alami et al., 2019). En revanche, les femmes vivant en milieu urbain, plus instruites et plus exposées à la modernité, sont plus enclines à faire vacciner leurs enfants que leurs consœurs du milieu rural (Akoto et al., 2002 ; Sagna, 2006). Les résultats de l'analyse explicative ne montrent pas de différence significative entre la vaccination des enfants résidents en milieu 
urbain et ceux résidents en milieu rural contrairement à ce qui a été observé au niveau descriptif. L'effet du milieu de résidence serait plutôt médiatisé par d'autres variables tel que le niveau d'instruction de la mère, le nombre de visites prénatales et le niveau de vie (Fournier et Haddad, 1995).

Bien que le taux de vaccination complète soit faible en Guinée, on observe des fortes disparités entre les régions de résidence. Les enfants de 12-23 mois sont relativement mieux vaccinés dans les régions de Kankan, Faranah et Nzérékoré. Ces résultats sont atypiques car on s'attend à ce que la couverture vaccinale soit plus grande dans la Capitale, Conakry et les régions voisines (Kindia et Boké) dénotant ainsi une meilleure corrélation entre le taux de vaccination et la disponibilité des services de santé. En effet, un rapport du Ministère de la Santé a montré qu'en 2014, que le ratio entre personnels soignants et population est de 0,35 en Guinée alors que la norme recommandée par l'OMS est 2,3. En outre, ce rapport indique que $83 \%$ des soignants résidaient en milieu urbain (principalement à Conakry), qui n'abrite que 30\% de la population du pays. On peut donc supposer que l'accroissement de la couverture vaccinale dans les régions de Kankan, Faranah et Nzérékoré, serait une conséquence de l'augmentation de personnel soignant intervenu dans ces régions à partir de 2015 suite à l'épidémie de la maladie à virus Ebola, et qui a obligé de renforcer la capacité des services et la disponibilité des soins de santé dans ces régions ${ }^{2}$. Cependant, on s'interroge sur le faible score de la région de Kindia qui a été l'une des plus touchées par l'épidémie d'Ebola après Nzérékoré.

Plusieurs études ont montré qu'un niveau socioéconomique faible des ménages est l'une des causes de la faible vaccination des enfants. Kalambayi et al. (2010) dans une étude en RDC ont indiqué que les enfants des ménages pauvres ont près de 2 fois moins de chance de ne pas être vaccinés que les enfants des ménages riches. Douba et al. (2015) ont trouvé un résultat similaire dans leur étude sur le Libéria, la Guinée et le Burkina. C'est le cas aussi de Ouedraogo et Diendéré (2004) au Burkina Faso, dans le district de Pissy, où ils ont constaté que les raisons de la non-vaccination des enfants étaient le niveau économique bas des mères.

\section{Conclusion}

En Guinée, en dépit des efforts consentis par le Gouvernement et ses partenaires, la couverture vaccinale demeure faible. Moins d'un quart des enfants de 12-23 mois (24\%) ont reçu tous les vaccins de base recommandés par l'OMS. Plusieurs facteurs expliqueraient cette situation : le faible niveau

Selon l'Annuaire statistique de la santé de 2013 et 2017, le ratio population par médecin a augmenté dans toutes les régions sauf à Conakry. A Boké l'augmentation est d'environ 1\%, $17 \%$ à Kindia, 24\% à Nzérékoré, 24\% à Mamou, 26\% à Labé, 34\% à Faranah et $44 \%$ Kankan. Tandis qu'à Conakry on note une forte baisse (-90\%). 
d'instruction de la mère, le faible recours des mères aux consultations prénatales et l'accouchement dans un établissement de santé, la pauvreté des ménages, la taille élevée du ménage, et l'effet propre de la région de résidence. D'autres facteurs qui n'étaient pas inclus dans les données tels que l'accessibilité géographique et financière et les perceptions sur les effets secondaires des vaccins pourraient avoir également des effets significatifs sur la vaccination complète des enfants (Coulibaly et al., 2008, Aka, 2010).

Les résultats obtenus peuvent servir à l'amélioration des stratégies du PEV pour l'augmentation de la couverture vaccinale des enfants, notamment par la sensibilisation des communautés sur l'importance de la vaccination des enfants et de leurs mères, la formation du personnel de santé en counseling afin de réduire les déperditions et le respect du calendrier de vaccination.

\section{References:}

1. Aalemi, A. K., Shahpar, K., \& Mubarak, M. Y. (2020). Factors influencing vaccination coverage among children age 12-23 months in Afghanistan: Analysis of the 2015 Demographic and Health Survey. PloS one, 15(8), e0236955. https://doi.org/10.1371/journal.pone.0236955.

2. Aka A., K. (2010). «L'accessibilité des populations rurales aux soins de santé dans le département d'Abengourou (Côte-d'Ivoire)». Les Cahiers d'Outre-Mer, 2010, n 251, p. 439-460. Bordeaux, France. https://journals.openedition.org/com/6075.

3. Akoto, E., Kouamé, A., \& Lamlenn, S. B. (2002). Se soigner aujourd'hui en Afrique de l'Ouest : pluralisme thérapeutique entre traditions et modernité (Bénin, Côte d'Ivoire et Mali) (No. 27). Institut de formation et de recherche démographiques, Yaoundé, Cameroun.

4. Adébiyi, G. B. (2011). Déterminants individuels et contextuels de la mortalité des enfants de moins de cinq ans en Afrique au sud du Sahara. Analyse comparative des enquêtes démographiques et de santé (Thèse de Doctorat en Démographie), Université de Montréal, Montréal, Canada. Consulté le 13/04/2020. https://core.ac.uk/download/pdf/55647727.pdf

5. Douba, A., Aka, L., Yao, G., Zengbé-Acray, P., Akani, B. \& Konan, N. (2015). Facteurs sociodémographiques associés à la vaccination incomplète des enfants de 12 à 59 mois dans six pays d'Afrique de l'ouest. Santé Publique, vol. 27(4), 575-584. https://doi.org/10.3917/spub.154.0575

6. Bbaale E. (2013). Factors influencing childhood immunization in Uganda. Journal of health, population, and nutrition, 31(1), 118-129. https://doi.org/10.3329/jhpn.v31i1.14756 
7. Baya, B. (1996). Les déterminants de la mortalité des enfants en milieu urbain au Burkina Faso: Cas de Bobo-Dioulasso.

8. Banza, B. (1998). Instruction des parents et survie de l'enfant au Burkina Faso: cas de Bobo-Dioulasso. Les dossiers du CEPED, 48, 27.

9. Bloom, D. E., Canning, D., \& Weston, M. (2005). The value of vaccination. WORLD ECONOMICS-HENLEY ON THAMES-, 6(3), 15.

10. Coulibaly I, Keita B., Kuepie M., (2008): «Les déterminants du recours thérapeutique au Mali : entre facteurs socioculturels, économiques et d'accessibilité géographique », Afrilux n³, 16.

11. Dixit, P., Dwivedi, L. K., \& Ram, F. (2013). Strategies to improve child immunization via antenatal care visits in India: a propensity score matching analysis. PloS one, 8(6), e66175.

12. Fournier, P., \& Haddad, S. (1995). Les facteurs associés à l'utilisation des services de santé dans les pays en développement. Sociologie des populations, 289, 325.

13. Gage, A. J., Sommerfelt, A. E., \& Piani, A. L. (1997). Household structure and childhood immunization in Niger and Nigeria. Demography, 34(2), 295-309.

14. Greenaway E.S, Leon J, Baker D.P (2012). Understanding the association between maternal education and use of health services in Ghana: exploring the role of health knowledge. J Biosoc Sci. ; 44(6), 733-47. doi: 10.1017/S0021932012000041.

15. Institut National de la Statistique (INS) et ICF International (2013). Enquête Démographique et de Santé et à Indicateurs Multiples (EDSMICS,2012. Conakry, Guinée, Calverton, Maryland, USA : INS et ORC Macro.

16. Institut National de la Statistique (INS) et ICF (2018): Enquête Démographique et de Santé en Guinée (EDS,2018). Conakry, Guinée, et Rockville, Maryland, USA : INS et ICF.

17. Ministère de la Santé (2014) : Rapport Général des Etats Généraux de la Santé en Guinée, Conakry, juin 2014.

18. Ndèye M., Papa N., Diédhiou A., Guèye A. S, Dia A. (2009) : «Facteurs d'abandon de la vaccination des enfants âgés de 10 à 23 mois à Ndoulo (Sénégal) » in Cahiers d'études et de recherches francophones / Santé. Volume 19, Numéro 1, 9-13. https://docplayer.fr/37669387-Etude-originale-facteurs-d-abandonde-la-vaccination.html

19. Ndonky A., Oliveau S. , Lalou R., Dos Santos S. (2015) : «Mesure de l'accessibilité géographique aux structures de santé dans l'agglomération de Dakar». European Geographique Journal. https://doi.org/10.4000/cybergeo.27312. 
20. OMS (1996). Situation mondiale des vaccins et vaccination. Genève : WHO/GPV/96.4.

21. OMS. Bulletin des maladies évitables par la vaccination. $\mathrm{n}^{\circ} 37$. NewYork : OMS ; AFRO, 2003. http://www.afro.who.int/en/africanvaccination-week/

22. OMS (2012) : Recommandations de l'OMS pour la vaccination systématique.

https://www.who.int/immunization/policy/routine_immunization_tab le_guidelines_fr.pdf?

23. OMS (2017) : Conférence Ministérielle sur la Vaccination en Afrique : tenir la promesse : assurer la vaccination pour tous en Afrique. https://www.afro.who.int/sites/default/files/2017-

06/Immunization+for+All+-+FR.pdf

24. OMS (2019) : «Les experts mettent en garde contre la stagnation de la couverture vaccinale en Afrique », Brazzaville, Congo, 23 janvier 2019, https://www.afro.who.int/fr/news/

25. ONU info : https://news.un.org/fr/story/2020/07/1073081

26. Programme élargi de vaccination, (2013) : Plan pluri annuel complet, 2011-2015. Ministère de la Santé et de l'Hygiène Publique, Conakry, Guinée.

https://extranet.who.int/countryplanningcycles/sites/default/files/coun try_docs/Guinea/ppac_guinee_version_finale_2014.pdf

27. Rakotondrabé Patricia (2004) : «Statut de la femme, prise de décision et santé des enfants à Madagascar », Thèse de doctorat, Institut de formation et de recherche démographiques (IFORD), Yaoundé, Cameroun.

28. Santoni F. (2001). Le Programme élargi de vaccination : 25 ans demain. Med Trop $2001 ; 1: 177-86$, cité par Ndèye et al.

29. Sawadogo S. (2008) : «Déterminants de la déperdition vaccinale au Burkina Faso », Mémoire de DESS, IFORD, Yaoundé, Cameroun.

30. Sia Drissa (20010) : "Stratégies et déterminants de la vaccination au Burkina Faso 1993 - 2003 », Thèse de doctorat en Santé Publique, Université de Montréal, Montréal, Canada, 2010. https://papyrus.bib.umontreal.ca/xmlui/bitstream/handle/1866/8852/S ia_Drissa_2010.pdf? sequence $=3$ \&isAllowed $=y$

31. Tefera, Y. A., A. L. Wagner, E. B. Mekonen, B. F. Carlson, and M. L. Boulton. 2018. "Predictors and Barriers to Full Vaccination among Children in Ethiopia." Vaccines (Basel) 6 (2).

32. Xeuatvongsa, A., M. Hachiya, S. Miyano, T. Mizoue, and T. Kitamura. 2017. "Determination of Factors Affecting the Vaccination Status of Children Aged 12-35 Months in Lao People's Democratic Republic." Heliyon 3 (3): e00265. https://doi.org/10.1016/j.heliyon.2017.e00265. 
33. Zoungrana C. (1993): «Déterminants socio-économiques de l'utilisation des services de santé maternelle et infantile à Bamako (Mali) », collection thèses et mémoires n³6, Université de Montréal, 214p. 